\title{
Expression of mitogen activated protein kinases in labial salivary glands of patients with Sjögren's syndrome
}

\author{
Hideki Nakamura, Atsushi Kawakami, Satoshi Yamasaki, Yojiro Kawabe, \\ Tatsufumi Nakamura, Katsumi Eguchi
}

\begin{abstract}
Objective-The expression of CD40 and CD40 ligand (CD40L) in mononuclear cells (MNCs) infiltrating the salivary glands of patients with Sjögren's syndrome (SS) has recently been reported. This study determined the expression of mitogen activated protein kinase (MAP kinase) superfamilies, which act as downstream effector molecules of CD40, in MNCs infiltrating labial salivary tissues in SS patients.
\end{abstract}

Methods-Six HTLV-I seronegative SS patients and 10 HTLV-I seropositive patients including five HTLV-I associated myelopathy (HAM) patients were examined. The expression of MAP kinase superfamilies in labial salivary glands was examined by immunohistochemistry containing the mirror section technique.

Results-Both active forms of c-Jun N-terminal kinase (JNK) and p38 were found in salivary infiltrating MNCs of SS patients. Only minimal expression of the active form of extracellular signal regulated kinase (ERK) was observed in these tissues, however, co-expression of active JNK and active p38 was confirmed by the mirror section technique. Furthermore, these protein kinases were co-expressed in CD $40^{+}$MNCs. No difference in expression levels of active JNK and p38 was found in patients who were positive or negative for anti-HTLV-I antibody.

Conclusion-These results indicate that JNK and p38, but not ERK, function as downstream effector molecules of CD40 in salivary infiltrating MNCs in SS patients, and suggest that these molecules may be involved in the pathological process of chronic sialadenitis in SS.

(Ann Rheum Dis 1999;58:382-385)

First Department of Internal Medicine, Nagasaki University School of Medicine, Nagasaki, Japan

Correspondence to: Professor K Eguchi, First Department of Internal Medicine, Nagasaki

University School of

Medicine, 1-7-1 Sakamoto,

Nagasaki City, Nagasaki

852-8501, Japan.

Accepted for publication 8 March 1999
Signalling through CD40 via its natural ligand CD40L stimulates cells and induces a variety of cellular events. Studies have shown that ligation of CD40 not only stimulates B cell proliferation, but apparently provides an essential signal for initiation of immunoglobulin isotype switching. ${ }^{1}$ The development of experimental allergic encephalomyelitis and lupus erythematosus in animal models is significantly suppressed by anti-CD40L monoclonal antibody $(\mathrm{mAb})$, flagging the importance of signals triggered by CD40 engagement. ${ }^{2-4}$ We recently described in situ expression of CD40/CD40L in salivary infiltrating mononuclear cells (MNCs) in patients with Sjögren's syndrome (SS). This may inhibit apoptotic processes in these cells, and instead promote $\mathrm{B}$ cell differentiation into plasma cells. ${ }^{5}$

CD40 receptor ligation activates mitogen activated protein (MAP) kinase superfamilies, especially c-JUN NH2 terminal kinases (JNK) and p38. ${ }^{6-8}$ Activation of $\mathrm{JNK}$ and p38 augments the transcriptional activity of Rel/ $\mathrm{NF}-\kappa \mathrm{B}$ and c-Jun/ATF-2, thus promoting expression of cellular genes such as cytokines and adhesion molecules. ${ }^{78}$ In addition, although the participation of the MAP kinase superfamilies is not clear, CD40 signalling renders the cells resistant to apoptotic stimuli with increment in $\mathrm{Bcl}-2$ or $\mathrm{Bcl}-\mathrm{xL}$ expression. ${ }^{910}$ Immunohistological studies have identified increased expression of various cytokines and adhesion molecules in the salivary glands of patients with SS. ${ }^{11}{ }^{12}$ Reduced apoptotic cell death in salivary infiltrating MNCs has also been identified. ${ }^{11}$ Therefore, we can speculate that in situ expression of CD40/CD40L in salivary infiltrating MNCs contributes to the pathological process of chronic sialadenitis in SS patients.

In this study, we examined the expression of MAP kinase superfamilies including extracellular signal regulated kinase (ERK), JNK, and p38 in labial salivary glands of patients with SS.

\section{Methods}

PATIENTS

Six HTLV-I seronegative (all women, aged 48.2 (SD 26.5) years, range: 19-86) and 10 HTLV-I seropositive SS patients were entered in this study. The latter group comprised five patients in whom SS was complicated by HTLV-I associated myelopathy (HAM), and five patients without HAM. The five HTLV-I seropositive, SS-HAM patients were all women aged 59.0 (SD 11.7) years (range: 40-72). The five HTLV-I seropositive patients without clinical manifestations of HAM were also all women, aged 54.0 (SD 15.1) years (range: 40-72). Patients with secondary SS complicated by other autoimmune diseases were not included in the study. All patients fulfilled the criteria for diagnosis of SS as defined by the European Community. ${ }^{13}$ In addition, three subjects who have sicca symptoms but cannot be diagnosed as SS by the European Community (histologically including no focus) were also examined in this study. Informed consent was obtained from all participating subjects 
Table 1 Immunohistochemical analysis of active $\mathfrak{F N K}, p 38$, and ERK expression

\begin{tabular}{llllll}
\hline & & Grade & fNK & p38 & ERK \\
\hline HTLV-I seronegative SS (n=6) & case 1 & 4 & + & + & - \\
& case 2 & 4 & + & \pm & - \\
& case 3 & 3 & + & + & - \\
& case 4 & 4 & + & + & - \\
& case 5 & 4 & ++ & ++ & \pm \\
HTLV-I seropositive SS (n=5) & case 6 & 3 & + & + & \pm \\
& case 1 & 3 & + & + & - \\
& case 2 & 4 & + & \pm & - \\
SS with HAM (n=5) & case 3 & 4 & + & + & - \\
& case 4 & 4 & + & + & \pm \\
& case 5 & 4 & + & \pm & - \\
& case 1 & 3 & + & + & - \\
Normal control & case 2 & 3 & + & \pm & - \\
& case 3 & 4 & + & + & - \\
& case 4 & 3 & + & \pm & - \\
& case 5 & 4 & + & + & - \\
& & & - & - & -
\end{tabular}

${ }^{\star}$ Grading defined by Chisholm and Mason $^{16}$; Presence of at least one focus of mononuclear cells per $4 \mathrm{~mm}^{2}$ section $=$ grade 3 . Percentage of positive cells; $(-): 0 \%,( \pm): 0-10 \%,(+): 10-50 \%,(++)$ $>50 \%$. Normal control: a representative case who has sicca symptoms but could not be diagnosed as SS. Note that JNK and p38 expressed in the salivary glands of all 16 SS patients. However, weak ERK expression was observed in only three of 16 SS patients $(18.8 \%)$. There was no expression of these three MAP kinases in normal subject.

and the study was conducted in accordance with human experimental guidelines of our institution.

BIOPSY OF LABIAL SALIVARY GLANDS

Biopsy specimens from minor labial salivary glands were obtained from the mucosa of the lower lip under local anaesthesia. Tissues were fixed in $4 \%$ paraformaldehyde (PFA) in phosphate buffered saline ( $\mathrm{pH}$ 7.4) immediately after biopsy and then immersed in sucrose. The tissues were then frozen in liquid nitrogen and stored at $-80^{\circ} \mathrm{C}$ until use.

MONOCLONAL AND POLYCLONAL ANTIBODIES USED FOR IMMUNOHISTOCHEMISTRY

Anti-CD40 mAb was purchased from Immunotech (Marseille Cedex, France). Polyclonal antibodies against the active forms of JNK and
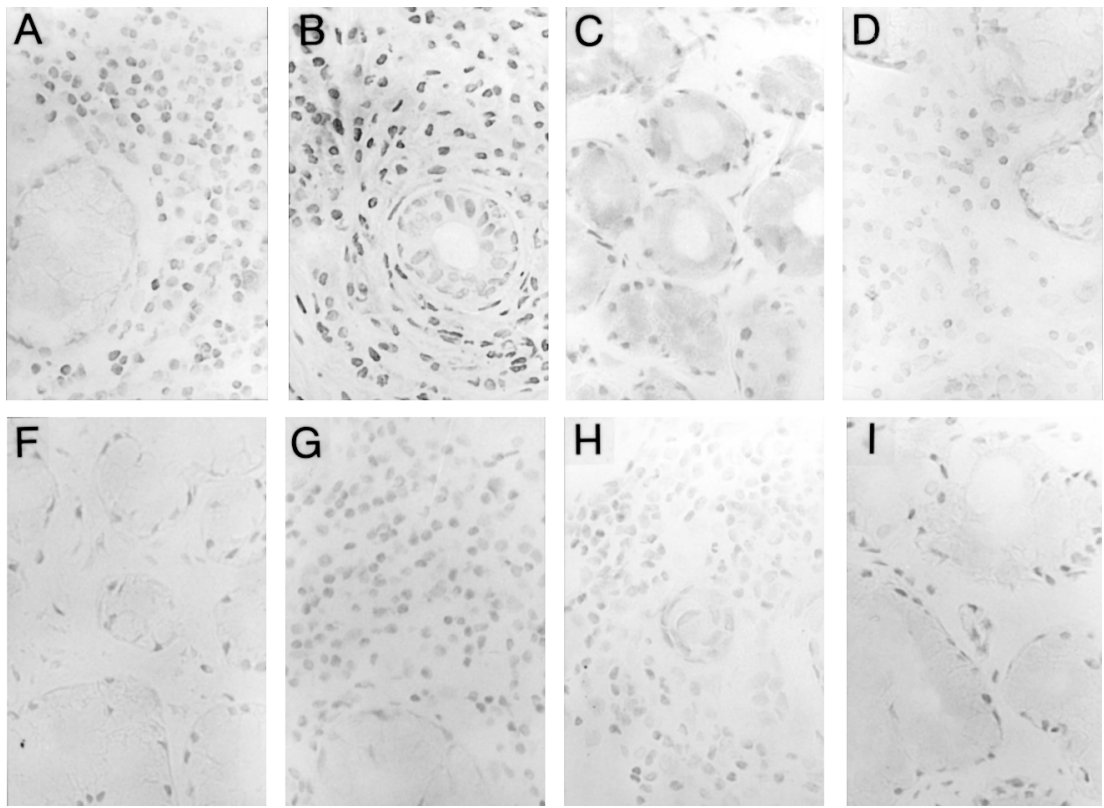

p38 were purchased from Promega Corporation (Madison, WI). Anti-active ERK (Thr202/ Tyr204) $\mathrm{mAb}$ was purchased from New England Biolabs, Inc (Beverly, MA).

\section{IMMUNOHISTOCHEMICAL EXAMINATION OF}

SALIVARY INFILTRATING MNCs

Tissue sections were cut and mounted on glass slides that were precoated with aminopropyltriethoxysilane. Sections were then stained by the labelled-streptavidin-biotin method (Histofine staining kit, Nichirei Co, Tokyo), as described previously. ${ }^{5}$ Briefly, endogenous peroxidase was inactivated in $3 \% \mathrm{H}_{2} \mathrm{O}_{2}$ solution and sections were then incubated with $10 \%$ goat or rabbit serum, followed by incubation with monoclonal and polyclonal antibodies for 60 minutes at room temperature. Sections were treated with biotinylated anti-rabbit or mouse IgG for 12 minutes. After washing, the sections were incubated with peroxidase conjugated streptavidin. Colour was developed using 3.3'diaminobenzidin and $\mathrm{H}_{2} \mathrm{O}_{2}$, and the slides were counterstained with methyl green. Negative control sections were treated with normal mouse IgG or normal rabbit serum. To make precise comparison of the same cells stained for two different proteins, two consecutive "mirror" sections were stained as previously described. ${ }^{5}$ The mirror sections were obtained using two consecutive sections mounted on the glass slides with the common cut surface facing upward, so that the same surface of the sectioned cells could be stained by two different antibodies. The percentage of positive cells was subjectively divided into four grades by an observer who was blinded to the type of tissue. The different grades included sections containing no positive cells $(-) ; 0-10 \%$ positive

Figure 1 Representative immunohistochemical analysis of $7 N K, p 38$ and ERK expression in serial sections of labial salivary glands from HTLV-I seropositive and seronegative SS patients. (A), (D), (G): HTLV-I seropositive SS patient. $(B),(E),(H)$ : HTLV-I seronegative SS patient. $(C),(F),(I)$ : Normal control (patient who have sicca symptoms but cannot to be diagnosed as $S S$ by the European Community). $(A),(B),(C)$ : active form of $\mathcal{F} N K$ expression. $(D),(E),(F)$ : active form of 38 expression. $(G),(H),(I)$ : active form of ERK expression. (F): Rheumatoid arthritis synovium (positive control for ERK expression in synovial cells; arrowhead). Note that both active forms of $7 N K$ and $p 38$ were found in SS patients with or without anti-HTLV-I antibody, however, neither $7 N K$ nor $p 38$ was found in normal control. Little active form of ERK was detected in labial salivary glands. (Original magnification $\times 400)$. 

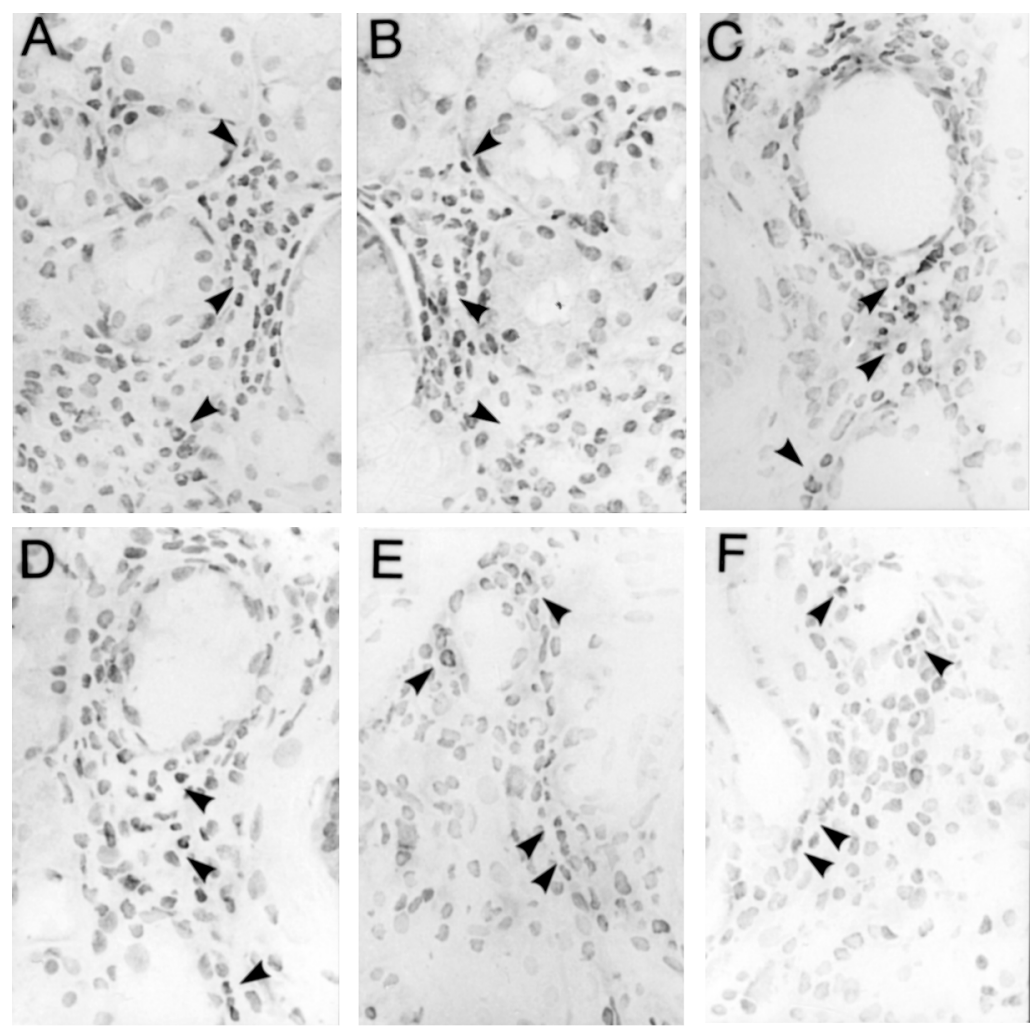

Figure 2 Co-expression of $7 N K-p 38, C D 40-7 N K$, and CD40-p38 in mirror sections of labial salivary glands from HTLV-I-seropositive SS patients. $(A),(B)$ : active form of $\mathcal{F N K}$ (A) and $p 38(B)$ were co-expressed in mirror sections in infiltrating $M N C s$ (arrowhead).
$(C),(D)$ : Co-expression of $C D 40$ (C) and active form of $\mathcal{F N K}(D)$ in infiltrating $M N C s$ (arrowhead). (E), (F): Co-expression of CD40 (E) and active form of $p 38(F)$ in infiltrating MNCs (arrowhead). (Original magnification $\times 400$ ).

cells $( \pm) ; 10-50 \%$ positive cells $(+)$, and $>50 \%$ positive cells $(++)$.

\section{Results}

EXPRESSION OF ACTIVE FORMS OF ERK, JNK AND P38 IN LABIAL SALIVARY GLANDS IN SS PATIENTS POSITIVE OR NEGATIVE FOR ANTI-HTLV-I ANTIBODY

We initially examined the expression of active forms of MAP kinase superfamilies in serial sections of labial salivary glands. As shown in figure 1, active forms of both $\mathrm{JNK}$ and p38 were detected in salivary infiltrating MNCs but not in acini or ducts. In contrast, minimal active ERK expression was evident in labial salivary glands of SS patients. Table 1 summarises active forms of JNK, p38 and ERK expression in all patients examined. We did not detect any difference in expression pattern between HTLV-I seropositive and HTLV-I seronegative SS patients.

CO-EXPRESSION OF JNK-P38, CD40-JNK, AND CD40-P38 IN LABIAL SALIVARY GLANDS OF PATIENTS WITH SS, CONFIRMED BY MIRROR SECTIONS

Active forms of $\mathrm{JNK}$ and p38 were expressed in the same infiltrating MNCs as determined by mirror sections (fig 2). Moreover, coexpression of CD40 with active forms of $\mathrm{JNK}$ or p38 was also confirmed (fig 2). These data strongly support the expression of active forms of both JNK and p38 in $\mathrm{CD}^{+} 0^{+}$salivary infiltrating MNCs in SS patients.

\section{Discussion}

Both $\mathrm{T}$ and $\mathrm{B}$ lymphocytes that infiltrate into the salivary glands of SS patients are activated, and express an activated phenotype. ${ }^{11}$ Expression of a variety of cytokines and adhesion molecules has been detected by immunohistological studies. ${ }^{112}$ These phenomena may contribute to the production of autoantibodies and serum hypergammaglobulinaemia, features of patients with SS. ${ }^{11}$

Precise molecular mechanisms that regulate the activation of salivary infiltrating MNCs in SS are not fully elucidated, however, one candidate molecular pathway is the CD40/ CD40L system, which is strongly expressed in these cells as determined by immunohistochemistry. ${ }^{5}$ In this study, we have demonstrated that active forms of $\mathrm{JNK}$ and $\mathrm{p} 38$, but not ERK, are expressed in salivary infiltrating MNCs in SS. A mirror section technique showed co-expression of the active forms of JNK and p38. Furthermore, the active forms of these stress activated protein kinases were co-expressed within $\mathrm{CD} 40^{+} \mathrm{MNCs}$. In vitro studies have shown that CD40 ligation rapidly and strongly activates MAP kinase superfamilies, especially JNK and p38..$^{6-8}$ This study suggests that both JNK and p38 are functional downstream molecules of CD40 signalling in situ in salivary infiltrating MNCs in SS.

We have previously demonstrated no difference in the expression of cell surface molecules, Fas/Fas ligand (FasL), and CD40/ CD40L in salivary infiltrating MNCs between HTLV-I seropositive and HTLV-I seronegative SS patients. ${ }^{514}$ Similarly, in this study, we could not detect any difference in the expression of ERK, JNK, and p38 between HTLV-I seropositive and HTLV-I seronegative SS patients, which shows the similarity of the pathological process inducing SS, irrespective of other associations with pathogenic organisms.

The active forms of both JNK and p38 are clearly expressed in salivary infiltrating MNCs in patients with SS. Our data suggest that signalling through CD40 to JNK and p38 may stimulate MNCs infiltrating the salivary glands of SS patients, activating nuclear transcriptional factors and therefore expression of cytokines and adhesion molecules that are intimately involved in the pathophysiology of SS.

Funding: this work was supported in part by a grant in aid (05670426) from the Ministry of Education, Science, Sport and Culture, Japan.

1 Stout RD, Suttles J. The many roles of CD40 in cell-mediated inflammatory responses. Immunol Today 1996; 17:487-92

Mohan C, Shi Y, Laman JD, Datta SK. Interaction between CD40 and its ligand gp39 in the development of murine lupus nephritis. J Immunol 1995;54:1470-80

Gerritse K, Laman JD, Noelle RJ, Aruffo A, Ledbetter JA Boersma WJA, et al. CD40-CD40 ligand interactions in experimental allergic encephalomyelitis and multiple sclerosis. Proc Natl Acad Sci USA 1996;93:2499-504.

4 Early GS, Zhao W, Burns CM. Anti-CD40 ligand antibody treatment prevents the development of lupus-like nephritis in a subset of New Zealand black $\mathrm{x}$ New Zealand white mice. J Immunol 1996;157:3159-64.

5 Nakamura H, Kawakami A, Tominaga M, Migita K, Kawabe Y, Nakamura T, et al. Expression of CD40/CD40 ligand and Bcl-2 family proteins in labial salivary glands of patients with Sjögren's syndrome. Lab Invest 1999;79: patients

6 Li YY, Baccam M, Waters SB, Pessin JE, Bishop GA, Koretzky GA. CD40 ligation results in protein kinase 
C-independent activation of ERK and JNK in resting murine splenic B cells. J Immunol 1996;157:1440-7.

7 Sutherland CL, Heath AW, Pelech SL, Young PR, Gold MR. Differential activation of the ERK, JNK, and p38 mitogen-activated protein kinases by CD 40 and the B cell antigen receptor. J Immunol 1996;157:3381-90

8 Craxton A, Shu G, Graves JD, Saklatvala J, Krebs EG, Clark EA. p38 MAPK is required for CD40-induced gen expression and proliferation in B lymphocytes. J Immunol 1998;161:3225-36.

9 Tsubata T, Wu J, Honjo T. B-cell apoptosis induced by antigen receptor crosslinking is blocked by a T-cell signal gen receptor crosslinking is blocked by

10 Ishida T, Kabayashi N, Tojo T, Ishida S, Yamamoto T, Inoue J. CD40 signaling-mediated induction of Bcl-xL Inoue J. CD40 signaling-mediated induction of

11 Sun D, Emmert-Buck MR, Fox PC. Differential cytokine mRNA expression in human labial minor salivary glands in primary Sjögren's syndrome. Autoimmunity 1998;28:12537.
12 Cauli A, Yanni G, Pitzalis C, Challacombe S, Panayi GS Cytokine and adhesion molecule expression in minor salivary glands of patients with Sjögren's syndrome. Ann Rheum Dis 1995;54:209-15.

13 Vitali C, Bombardieri S, Moutsopoulos HM, Balestrieri G Bencivelli W, Bernstein RM, et al. Preliminary criteria for the classification of Sjögren's syndrome: results of a prospective concerted action supported by European Community. Arthritis Rheum 1993;36:340-7.

14 Nakamura H, Eguchi K, Nakamura T, Mizokami A, Shirabe S, Kawakami A, et al. High prevalence of Sjögren's syndrome in patients with HTLV-I associated myelopathy. Ann Rheum Dis 1997;56:167-72.

15 Nakamura H, Koji T, Tominaga M, Kawakami A, Migita K, Kawabe Y, et al. Apoptosis in labial salivary glands from Sjögren's syndrome patients.- comparison with the Sjögren's syndrome patients.- comparison, with the
HTLV-I seronegative and -seropositive Sjögren's syndrome patients. Clin Exp Immunol 1998;104:106-12.

16 Chisholm DN, Mason DK. Labial salivary gland biopsy in Sjögren's disease. J Clin Pathol 1968;21:656-60. 